\title{
Decoherent Histories and Quantum State Diffusion
}

\author{
LAJos Diósi \\ KFKI Research Institute for Particle and Nuclear Physics \\ H-1525 Budapest 114, POB 49 \\ HUNGARY
}

NiCOLAS Gisin

Group of Applied Physics, University of Geneva

1211 Geneva 4

SWITZERLAND

JONATHAN HALLIWELL

Theory Group, Blackett Laboratory

Imperial College, London SW7 2BZ

$U K$

and

IAN C. Percival

Department of Physics, Queen Mary and Westfield College

Mile End Road, London E1 4NS

$U K$

PACS Numbers: 03.65.-w, 03.65.Bz, 05.40.+j, 42.50.-p

Preprint IC 93-94/25. March, 1994

Submitted to Physical Review Letters

\begin{abstract}
We demonstrate a close connection between the decoherent histories $(\mathrm{DH})$ approach to quantum mechanics and the quantum state diffusion (QSD) picture, for open quantum systems described by a master equation of Lindblad form. The (physically unique) set of variables that localize in the QSD picture also define an approximately decoherent set of histories in the DH approach. The degree of localization is related to the degree of decoherence, and the probabilities for histories prescribed by each approach are essentially the same.
\end{abstract}


The last decade has witnessed considerable interest in the foundations of quantum mechanics. Many reasons for this may be found: the long-felt dissatisfaction with the Copenhagen interpretation; certain experimental developments and the Copenhagen interpretation's inability to supply a useful qualitative account of them; the special needs of quantum mechanics applied to the entire universe (quantum cosmology); and the general desire to possess a deeper understanding of quantum theory. Partially for these reasons, modifications and generalizations of both the mathematics and interpretation of quantum theory have been sought.

This letter is concerned with demonstrating the connections between two recently developed alternative approaches to quantum theory, each of which was proposed, independently, with the aim of shedding light on some of the difficulties outlined above. The approaches we shall compare are the decoherent (or "consistent") histories approach $[1,2]$ and the quantum state diffusion picture $[3,4,5]$ (see Ref.[6] for an early guess at the relation between these two approaches). In both of these approaches, the basic mathematical formalism of quantum mechanics is left untouched, but new insight into its interpretation is obtained by focusing on different types of mathematical objects.

We will be concerned with a quantum system consisting of a subsystem coupled to its environment. The subsystem is then frequently referred to as an open quantum system, and we shall do so here. Conventionally, an open system is described by a reduced density operator $\rho$, evolving according to a master equation, derived by tracing over the environment. Under the assumption that the evolution is Markovian, the master equation takes the Lindblad form [7],

$$
\frac{d \rho}{d t}=-\frac{i}{\hbar}[H, \rho]-\frac{1}{2} \sum_{j=1}^{n}\left(\left\{L_{j}^{\dagger} L_{j}, \rho\right\}-2 L_{j} \rho L_{j}^{\dagger}\right)
$$

Here, $H$ is the Hamiltonian of the open system in the absence of the environment (sometimes modified by terms depending on the $L_{j}$ ) and the $n$ operators $L_{j}$ model the effects of the environment. This equation is frequently used in quantum optics [8] and in studies of decoherence $[9,10]$. For example, in the much-studied quantum Brownian motion model $[11,12,13]$, the master equation is (1) with a single Lindblad operator 
$L=(2 D)^{-\frac{1}{2}}\left(\hat{x}+2 \frac{i}{\hbar} \gamma D \hat{p}\right)$, with $D=\hbar^{2} /(8 m \gamma k T)$ (where $\gamma$ is the dissipation and $T$ is the temperature of the environment), and $H=H_{S}+\frac{1}{2} \gamma\{\hat{x}, \hat{p}\}$, where $H_{S}$ is the distinguished subsystem Hamiltonian, in the absence of the environment.

Density operators satisfying (1) give statistical predictions in full agreement with experiment in a wide variety of situations. However, they do not give a picture of the behaviour of an individual system, but only of ensembles. The quantum state diffusion approach avoids this shortcoming $[3,4,5,14]$. It originated from considerations of the quantum measurement problem $[15,16,17,18]$, and the desiderata of describing individual experimental outcomes and putting physical intuition into the equations, as urged by Bell [19]. It was also motivated by its computational advantage and insight in treating practical problems in open systems [20]. It consists of an "unravelling" of the evolution of $\rho$ under (1). This involves regarding $\rho$ as a mean over a distribution of pure state density operators, $\rho=M|\psi\rangle\langle\psi|$, where $M$ denotes the mean (defined below), with the pure states evolving according to the non-linear stochastic Langevin-Ito equation,

$$
\begin{aligned}
|d \psi\rangle=-\frac{i}{\hbar} H|\psi\rangle d t & +\frac{1}{2} \sum_{j}\left(2\left\langle L_{j}^{\dagger}\right\rangle L_{j}-L_{j}^{\dagger} L_{j}-\left\langle L_{j}^{\dagger}\right\rangle\left\langle L_{j}\right\rangle\right)|\psi\rangle d t \\
& +\sum_{j}\left(L_{j}-\left\langle L_{j}\right\rangle\right)|\psi\rangle d \xi_{j}(t)
\end{aligned}
$$

for the normalized state vector $|\psi\rangle$. Here, the $d \xi_{j}$ are independent complex differential random variables representing a complex Wiener process. Their linear and quadratic means are, $M\left[d \xi_{j} d \xi_{k}^{*}\right]=\delta_{j k} d t, M\left[d \xi_{j} d \xi_{k}\right]=0$ and $M\left[d \xi_{j}\right]=0$.

The quantum state diffusion picture described by the Ito equation (2) is mathematically equivalent to the Lindblad equation (1). However, its appeal lies in the fact that the solutions to the Ito equation appear to correspond rather well to individual experimental runs, and thus provide considerable insight into the behaviour of individual processes and systems. Solutions to the Ito equation commonly have the property of localization - the dispersion of certain operators tends to decrease as time evolves. This has been demonstrated by numerical solutions [5], analytic solutions in special cases [13,18,21], and some general theorems $[4,14]$. The method has also been successfully used to analyze quantum jump experiments [22]. 
Our first results, required for the comparison with the decoherent histories approach below, are explicit representations of the solutions to the Lindblad equation (1) and the Ito equation (2). To solve the Lindblad equation, consider the case of a single Lindblad operator $L=L_{R}+i L_{I}$, where $L_{R}, L_{I}$ are hermitian. Divide the finite time interval [0,t] into $K$ subintervals, so that $t=K \delta t$, and let $\delta t \rightarrow 0, K \rightarrow \infty$, holding $t$ constant. Then we have the following representation solution to (1):

$$
\begin{aligned}
\rho(t) & =\lim _{\delta t \rightarrow 0, K \rightarrow \infty}\left(\frac{\delta t}{\pi}\right)^{K} \int d^{2} \ell_{1} \cdots d^{2} \ell_{K} \\
& \times \prod_{m=1}^{K} \exp \left(\frac{\delta t}{2}\left(\ell_{m}^{*} L-\ell_{m} L^{\dagger}\right)\right) \exp \left(-\frac{\delta t}{2}\left|L-\ell_{m}\right|^{2}\right) \exp \left(-\frac{i}{\hbar} H_{0} \delta t\right) \rho(0) \\
& \times \prod_{m=1}^{K} \exp \left(\frac{i}{\hbar} H_{0} \delta t\right) \exp \left(-\frac{\delta t}{2}\left|L-\ell_{m}\right|^{2}\right) \exp \left(-\frac{\delta t}{2}\left(\ell_{m}^{*} L-\ell_{m} L^{\dagger}\right)\right)
\end{aligned}
$$

where $H_{0}=H+\frac{i \hbar}{4}\left[L, L^{\dagger}\right]$, and the $\ell_{m}$ are complex numbers at the discrete moments of time labeled by $m$. We use the notation $\left|L-\ell_{m}\right|^{2} \equiv\left(L_{R}-\operatorname{Re} \ell_{m}\right)^{2}+\left(L_{I}-\operatorname{Im} \ell_{m}\right)^{2}$. That this is the solution is readily verified by explicit computation [23]. The solution has the form of a "measurement process" of the $L$ 's, continuous in time, with "feedback" via the terms $\left(\ell_{m}^{*} L-\ell_{m} L^{\dagger}\right)[24]$. The case of many different Lindblad operators, $L_{j}$, is readily obtained by taking products over $j$ of the appropriate operators in (3) at each moment of time. The ordering of the operators at each moment of time is irrelevant in the limit $\delta t \rightarrow 0$ (although the operators at different times are time-ordered, according to increasing m). For future reference we write Eq.(3) in terms of a density operator propagator as $\rho(t)=K_{0}^{t}[\rho(0)]$.

Similarly, the solution to the Ito equation has the explicit representation

$$
\begin{aligned}
|\psi(t)\rangle=\lim _{\delta t \rightarrow 0} \prod_{m=1}^{K} & \exp \left(\frac{\delta t}{2}\left(2\left\langle L^{\dagger}\right\rangle_{m} L-L^{\dagger} L-\left\langle L^{\dagger}\right\rangle_{m}\langle L\rangle_{m}\right)+\left(L-\langle L\rangle_{m}\right) \delta \xi\right) \\
& \times \exp \left(-\frac{i}{\hbar} H \delta t\right)|\psi(0)\rangle .
\end{aligned}
$$

where $\langle L\rangle_{m}$ denotes the expectation of $L$ at time $t_{m}=m \delta t$. Eq.(4) expresses an individual history $\left|\psi_{\xi}(t)\right\rangle$ as an explicit functional of an individual complex gaussian noise $\xi(t)$. However, to make clearer the connection with Eq.(3) (and ultimately with the decoherent 
histories approach discussed below), it is useful to present an alternative representation, obtained by exchanging the Wiener process $\xi(t)$ for the stochastic variable $\ell(t)$, defined by $(\ell(t)-\langle L\rangle) d t=d \xi^{*}(t)($ Ref.[18]). Eq.(4) thus becomes

$$
\begin{aligned}
& |\psi(t)\rangle=\lim _{\delta t \rightarrow 0, K \rightarrow \infty}\left(\frac{\delta t}{\pi}\right)^{K / 2} N\left(\ell_{1} \cdots \ell_{K}\right) \\
& \times \prod_{m=1}^{K} \exp \left(\frac{\delta t}{2}\left(\ell_{m}^{*} L-\ell_{m} L^{\dagger}\right)\right) \exp \left(-\frac{\delta t}{2}\left|L-\ell_{m}\right|^{2}\right) \exp \left(-\frac{i}{\hbar} H_{0} \delta t\right)|\psi(0)\rangle
\end{aligned}
$$

where $H_{0}$ is given above after Eq.(3). $N$ is a (complex) normalization factor, ensuring that $\langle\psi(t) \mid \psi(t)\rangle=1$, and appears because the state is now to be thought of as a functional of the stochastic process $\ell(t)$ instead of $\xi(t),|\psi\rangle=\left|\psi_{\ell}\right\rangle$. Again the generalization to many Lindblad generators is straighforward. That (4), (5) are solutions to Eq.(2) may be verified by explicit computation [23]. This explicit representation of the solution clearly indicates that the solutions will tend to exhibit localization in the operator $L$. It also illustrates that the solutions have the form of a "trajectory", or "history", concentrated around the $\ell(t)$ corresponding to the particular Wiener process $\xi(t)$.

We may associate a probability with these trajectories. To see this, recall that we are working from the outset with a statistical ensemble of solutions to the Ito equation, $\left|\psi_{\xi}\right\rangle$, obeying the rules of standard ("classical") probability theory, and the probability distribution of the solutions is that implied by the means of $d \xi$, etc., given above. Indeed, the solution (3) to the master equation, is a sum over $\xi$ of $\left|\psi_{\xi}\right\rangle\left\langle\psi_{\xi}\right|$, weighted by the probability for each solution. As before, it is more useful to express this probability distribution as a probability $p[\ell(t)]$ over the states $\left|\psi_{\ell}\right\rangle$ satisfying Eq.(5). Since Eq.(3) must be a mean over $\left|\psi_{\ell}\right\rangle\left\langle\psi_{\ell}\right|$, it is easily seen from Eqs.(3) and (5), that the probability distribution over the $\left|\psi_{\ell}\right\rangle$ 's must be $p[\ell(t)]=|N[\ell(t)]|^{-2}$ (or what amounts to the same, the norm of the state (5) but without the normalization factor $N$ ). Note, however, that in general $\left\langle\psi_{\ell^{\prime}} \mid \psi_{\ell}\right\rangle \neq 0$ for $\ell \neq \ell^{\prime}$, and thus $p[\ell(t)]$ may be thought of as a probability for histories of values of $L$ only when the solutions $\left|\psi_{\ell}\right\rangle$ are reasonably well-localized in $L$.

The Lindblad and Ito equations, (1), (2), are invariant under redefinitions of the $L_{j}$ 's by unitary transformations, i.e., $L_{j} \rightarrow L_{j}^{\prime}=\sum_{k=1}^{n} U_{j k} L_{k}$, where $U_{k j}$ is a unitary matrix. 
For the case $n=1$, this is just multiplication by a constant phase, and the solutions (3), (4), do indeed manifest this invariance [3].

Turn now to the decoherent histories approach. The decoherent histories approach is a generalization of quantum mechanics to genuinely closed systems, such as the entire universe $[1,2]$. Its aim is to give a predictive formulation of quantum theory applicable to closed systems which does not rely on notions of measurement or on the existence of an external classical domain. From such a framework, one hopes to understand the emergence of the classical world from an underlying quantum one, and the origin of the quantum-classical division upon which the Copenhagen interpretation depends.

In the decoherent histories approach, the mathematical objects one focuses on are the probabilities for histories of a closed system. A quantum-mechanical history is defined by an initial state $\rho$ at time $t=0$ together with a string of projection operators $P_{\alpha_{1}} \cdots P_{\alpha_{n}}$ acting at times $t_{1} \ldots t_{n}$, characterizing the possible alternatives of the system at those times. The projections are exhaustive, $\sum_{\alpha} P_{\alpha}=1$, and exclusive, $P_{\alpha} P_{\beta}=\delta_{\alpha \beta} P_{\alpha}$. Because of interference, most sets of histories for a closed system cannot be assigned probabilities. The interference between pairs of histories in a set is measured by the so-called decoherence functional,

$$
D\left(\underline{\alpha}, \underline{\alpha}^{\prime}\right)=\operatorname{Tr}\left(P_{\alpha_{n}}\left(t_{n}\right) \cdots P_{\alpha_{1}}\left(t_{1}\right) \rho P_{\alpha_{1}^{\prime}}\left(t_{1}\right) \cdots P_{\alpha_{n}^{\prime}}\left(t_{n}\right)\right)
$$

where $P_{\alpha_{k}}\left(t_{k}\right)=e^{-\frac{i}{\hbar} H t_{k}} P_{\alpha} e^{\frac{i}{\hbar} H t_{k}}, H$ is the Hamiltonian of the closed system and $\underline{\alpha}$ denotes the string $\alpha_{1} \cdots \alpha_{n}$. When $D\left(\underline{\alpha}, \underline{\alpha}^{\prime}\right) \approx 0$ for $\underline{\alpha} \neq \underline{\alpha}^{\prime}$, inteference may be neglected, and one may assign the probability $p(\underline{\alpha})=D(\underline{\alpha}, \underline{\alpha})$ to the history. Probabilities assigned under this condition may be shown to obey the sum rules of probability theory [1]. Sets of histories satisfying this condition are called decoherent. Loosely, satisfaction of these conditions means that one can "talk about" (i.e., apply classical logic to) the physical properties of the system, and think about those properties as if they were definite, without having to invoke notions of measurement. Given the Hamiltonian and initial state for a closed system, one's initial aim is to determine the strings of projection operators for which the decoherence condition is satisfied. 
The decoherent histories approach is readily applied to the class of open systems con-

sidered here, i.e., closed systems in which there is a natural separation into a distinguished subsystem and the rest. For such systems, a natural set of histories to study are those characterized by the properties of the distinguished subsystem at each moment of time, but ignoring (i.e., coarse-graining over) the properties of the environment. Histories of this type are often decoherent as a result of the interaction between the system and the environment. To be precise, consider histories characterized by strings of projections $P_{\alpha_{1}} \otimes I^{\mathcal{E}} \ldots P_{\alpha_{n}} \otimes I^{\mathcal{E}}$ at times $t_{1} \cdots t_{n}$, where $I^{\mathcal{E}}$ denotes the identity on the environment. Now assuming that the initial density operator factorizes, the trace over the environment may be carried out explicitly in the decoherence functional (6), and, in the regime in which a Markovian approximation holds, it then has the form

$$
D\left(\underline{\alpha}, \underline{\alpha}^{\prime}\right)=\operatorname{Tr}\left(P_{\alpha_{n}} K_{t_{n-1}}^{t_{n}}\left[P_{\alpha_{n-1}} \cdots K_{t_{1}}^{t_{2}}\left[P_{\alpha_{1}} K_{0}^{t_{1}}[\rho(0)] P_{\alpha_{1}^{\prime}}\right] \cdots P_{\alpha_{n-1}^{\prime}}\right]\right)
$$

Here, $K_{t_{k}}^{t_{k+1}}$ is the reduced density operator propagator introduced above, and the trace is now over the distinguished subsystem only.

Given this expression, and given the explicit form of $K$ above, Eq.(3), we may now discuss decoherence. For simplicity, consider the case of projections continuous in time in the decoherence functional (7). The discrete time version of the decoherence functional will contain terms of the form,

$$
P_{\alpha_{k}} K_{t_{k-1}}^{t_{k}}[\cdots] P_{\alpha_{k}^{\prime}}=\int d^{2} \ell P_{\alpha_{k}} \exp \left(-\frac{\delta t}{2}|L-\ell|^{2}\right)[\cdots] \exp \left(-\frac{\delta t}{2}|L-\ell|^{2}\right) P_{\alpha_{k}^{\prime}}
$$

The operator $\exp \left(-\frac{\delta t}{2}|L-\ell|^{2}\right)$ is an approximate projection operator in the limit, used here, of small $\delta t$. (It is an approximate projector for all $\delta t$ if $\left[L, L^{\dagger}\right]=0$ ). Now the key point is that on the right hand side, we have two different projection operators $P_{\alpha_{k}}$, $P_{\alpha_{k}^{\prime}}$ operating on the same Gaussian projection, $\exp \left(-\frac{\delta t}{2}|L-\ell|^{2}\right)$. Because Gaussian projections are approximately exclusive, the decoherence functional will be approximately diagonal in the $\alpha_{k}$ 's if we choose the projections $P_{\alpha_{k}}$ also to be Gaussian projections onto $L:$

$$
P_{\alpha}=\exp \left(-\frac{1}{2} \kappa^{2} \delta t\left|L-\kappa^{-1} \delta t^{-\frac{1}{2}} \alpha\right|^{2}\right)
$$


Here, $\alpha$ is a dimensionless and complex continuous label, and $\kappa^{-1}(\delta t)^{-\frac{1}{2}}$ is the width of the projection which will be tuned by the dimensionless parameter $\kappa$. The approximate exclusivity of these approximate projectors means that $\alpha$, although continuous, has significance only up to order 1 . Clearly, with the choice (9), (8) will be very small unless $\alpha \approx \kappa \delta t^{\frac{1}{2}} \ell \approx \alpha^{\prime}$. Therefore, histories characterized by strings of projections onto $L$ will approximately decohere. We thus arrive at our main result: the variables exhibiting localization in the quantum state diffusion picture are the same as the variables characterizing a decoherent set of histories in the decoherent histories approach.

The operator (9) is an approximate projection operator (under the conditions stated above) onto a subset of the spectrum of the (generally non-hermitian) operator $L$, and the label $\alpha$ is complex. Projections of precisely this type have not previously been used in the decoherent histories approach, but there is no obvious obstruction to doing so. Indeed, the use of such projections is strongly suggested by form of Eq.(8), which arises as a result of the invariance of Eq.(3) under $L \rightarrow e^{i \phi} L$. Furthermore, the connection with histories characterized by the more familiar types of projectors may be made by specialization to the case of a hermitian $L$ (strictly, to the class of operators equivalent to a hermitian operator under multiplication by a phase).

The degrees of localization and decoherence also are related. From the solution to the Ito equation (5), the degree of localization is determined by the degree to which $L$ becomes concentrated about a particular trajectory $\ell(t)$. At each time step $\delta t$, the localization width of $L$ is of order $\delta t^{-\frac{1}{2}}$. Similarly, from equation (8), one may see that the degree of decoherence is also determined by the degree to which $L$ is concentrated about a particular value. Loosely, the off-diagonal terms of the decoherence functional are suppressed in comparison to the on-diagonal terms (this is the appropriate way to measure approximate decoherence [2]) by a factor of order of $\exp \left(-\kappa^{-2}\left|\alpha-\alpha^{\prime}\right|^{2} \times\right.$ constant $)$, where the constant is of order 1. This means that the projectors given by Eq.(9) define an approximately decoherent set of histories only if $\kappa<<1$.

The degrees of localization and decoherence are related, therefore, in the sense that approximate decoherence of histories may be achieved only if the projectors $P_{\alpha}$ characterizing 
the histories are coarser than the localization width.

Finally, consider the probabilities for histories. Given approximate decoherence, the decoherent histories approach assigns probabilities to histories given by the diagonal elements of the decoherence functional (7). Consider Eq.(8), but now with $\alpha_{k}=\alpha_{k}^{\prime}$. Under the conditions yielding approximate decoherence, the integrand in (8) will be very small unless $\ell \approx \kappa^{-1}(\delta t)^{-\frac{1}{2}} \alpha$. The projection operators $P_{\alpha_{k}}$ then have essentially no effect (except to produce a negligible modification of the width of the neighbouring Gaussian projectors) so we can drop them. One thus finds that

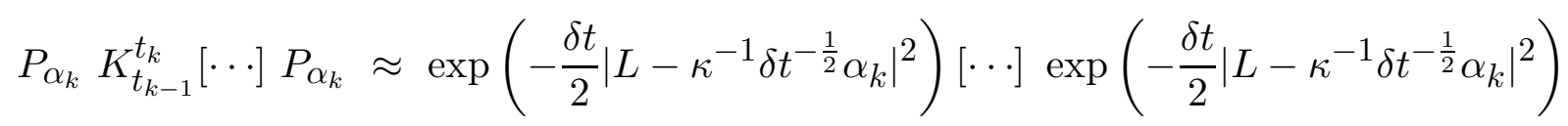

Using this result, one may then see that the probabilities assigned to these histories in the decoherent histories approach have the form of the norm of the state (5) without the

normalization factor, and with $\ell\left(t_{k}\right)=\kappa^{-1} \delta t^{-\frac{1}{2}} \alpha\left(t_{k}\right)$. They are therefore exactly the same as the probabilities assigned in the quantum state diffusion approach. A more detailed account of this work will be presented elsewhere [23]

Acknowledgements: L.D. thanks support by the grant OTKA No. 1822/1991. J.J.H. was supported by the Royal Society.

\section{REFERENCES}

1. M.Gell-Mann and J.B.Hartle, Phys.Rev. D47, 3345 (1993); R.Griffiths, J.Stat.Phys. 36, 219 (1984); R.Omnès, Rev.Mod.Phys. 64, 339 (1992).

2. H.F.Dowker and J.J.Halliwell, Phys.Rev. D46, 1580 (1992)

3. N. Gisin and I.C. Percival, J.Phys. A25, 5677 (1992); see also Phys. Lett. A167, 315 (1992).

4. N. Gisin and I.C.Percival, J.Phys. A26, 2233 (1993).

5. N. Gisin and I.C. Percival, J.Phys. A26, 2245 (1993). 
6. L.Diósi, Phys.Lett. B280, 71 (1992).

7. G.Lindblad, Comm.Math.Phys. 48, 119 (1976).

8. H.J.Carmichael, An Open Systems Approach to Quantum Optics (Springer-Verlag, 1993).

9. W.H. Zurek, Physics Today 40, 36 (1991); J.P.Paz, S.Habib and W.H.Zurek, Phys.Rev. D47, 488 (1993).

10. H.D. Zeh, Phys. Lett. A172, 189 (1993); E.Joos and H.D.Zeh, Zeit.Phys. B59, 223 (1985). See also N. Gisin and I. Percival, Phys. Lett. 175A, 144 (1993).

11. A.O.Caldeira and A.J.Leggett, Physica 121A, 587 (1983).

12. L.Diósi, Physica A199, 517 (1993).

13. Y.Salama and N.Gisin, Phys. Lett. 181A, 269, (1993).

14. I.C.Percival, J.Phys. A27, 1003 (1994).

15. P.Pearle, Phys.Rev. D13, 857 (1976).

16. N.Gisin, Phys.Rev.Lett. 52, 1657 (1984); Hevl.Phys.Acta 62, 363 (1989).

17. L.Diósi, J.Phys. A21, 2885 (1988)

18. L.Diósi, Phys.Lett. 129A, 419 (1988).

19. J.S.Bell, Physics World 3, 33 (1990).

20. I.Percival, in Quantum Chaos and Quantum Measurement, NATO Advanced Study Institute Series, Vol 357, edited by P.Cvitanovic et al. (Kluwer, Dordrecht,1992), p.199.

21. L.Diósi, Phys.Lett 132A, 233 (1988).

22. N. Gisin, P.L. Knight, I.C. Percival, R.C. Thompson and D.C. Wilson, J. Mod. Optics, 40, 1663 (1993); B.Garraway and P.Knight, Phys.Rev. A49, 1266 (1994); P.Goetsch and R.Graham, Ann.Physik 2, 706 (1993). 
23. L.Diósi, N.Gisin, J.Halliwell and I.Percival, in preparation.

24. C.M.Caves and G.J.Milburn, Phys.Rev. A36, 5543 (1987). 